BI-TP 2006/12

CERN-PH-TH/2006-077

\title{
Moduli decay in the hot early Universe
}

\author{
Dietrich Bödeker ${ }^{1}$ \\ Physics Dept., Theory Unit, CERN, CH-1211 Geneva 23, Switzerland \\ and \\ Fakultät für Physik, Universität Bielefeld, D-33615 Bielefeld, Germany ${ }^{2}$
}

\begin{abstract}
We consider moduli fields interacting with thermalized relativistic matter. We determine the temperature dependence of their damping rate and find that it is dominated by thermal effects in the high temperature regime, i.e. for temperatures larger than their mass. For a simple scalar model the damping rate is expressed through the known matter bulk viscosity. The high temperature damping rate is always smaller than the Hubble rate, so that thermal effects are not sufficient for solving the cosmological moduli problem.
\end{abstract}

\footnotetext{
${ }^{1}$ e-mail: bodeker@physik.uni-bielefeld.de

${ }^{2}$ Permanent address
} 
Many theories beyond the Standard Model of particle physics, such as string and supersymmetric theories, contain directions in field space that have a flat potential in the supersymmetric limit and couple to other fields only through Planck scale suppressed interactions. If these directions are stabilized by the same mechanism which is responsible for supersymmetry breaking, a particle whose mass is of the order of a $\mathrm{TeV}$ and a dangerously large lifetime may result. Such fields are often called moduli. If one of the moduli starts at a value displaced from the minimum of the effective potential, it will perform coherent oscillations around its minimum. The energy of these oscillations decreases like the energy density in non-relativistic matter with the expansion of the Universe, $\rho \propto a^{-3}$, while for relativistic matter $\rho \propto a^{-4}$. Therefore the moduli may soon contribute significantly to the total energy density of the Universe.

In the vacuum the decay rate of a moduli field with mass $m$ is of order $m^{3} / M^{2}$, where $M=2 \times 10^{18} \mathrm{GeV}$ is the reduced Planck mass [1]. The corresponding long lifetime $\tau=\Gamma^{-1} \sim 10^{5}(\mathrm{TeV} / m)^{3}$ s causes the cosmological moduli problem [1]- 6 ]. Unless the initial amplitude of the moduli is very small, they dominate the energy density of the Universe at some point, spoiling the success of standard big bang nucleosynthesis.

Many solutions to this problem have been suggested. Recently it was pointed out in Ref. [7] that in the hot early Universe the moduli oscillations could be damped much more strongly than in the vacuum. It was found that the damping is in fact so strong that the moduli would adiabatically follow the minimum of the effective potential, making their relic density harmless for cosmology [7]. In this note we reconsider the thermal damping of scalar moduli fields. Unfortunately, we cannot confirm the result of Ref. 7].

A modulus field starts oscillating once the Hubble rate $H$ drops below its mass $m$, which happens at the temperature $T_{\text {osc }} \sim \sqrt{m M} \sim 10^{10} \mathrm{GeV} \sqrt{m / \mathrm{TeV}}$. The thermal corrections to the mass have a magnitude $\lesssim T^{2} / M$, which is of the same order as $H$ if the Universe is radiation-dominated. Thus, unless they come with a very large coefficient [8], they do not affect the parametric size of $T_{\text {osc }}$, and they can be neglected for $T \ll T_{\text {osc }}$.

Thermal damping effects can become important if the damping rate becomes larger than the Hubble rate. In order to see whether this happens, we can neglect the expansion of the Universe. We consider temperatures $T \gg m$, because, as we shall see, only in this case does thermal damping dominate over the vacuum damping. Then the matter fields, i.e. fields that have unsuppressed interactions, evolve on time scales of order $T^{-1}$, while the modulus field evolves much more slowly. 
To describe the time evolution of this non-equilibrium system, we imagine starting from thermal equilibrium at $t=-\infty$. For simplicity the zero of the modulus field $\varphi$ is chosen such that its expectation value at the considered temperature vanishes. To drive the system out of equilibrium we add the term $\mathcal{L}_{\text {ext }}=j \varphi$ to the Lagrangian, where $j$ is an external c-number field that vanishes for $t \rightarrow-\infty$. Then, at some finite time, $j$ is switched off again. From then on we have an isolated system which is not in thermal equilibrium. The way the system got out of equilibrium does not matter because $\varphi$ evolves much more slowly than all other degrees of freedom, since $m \ll T$. On time scales over which $\varphi$ changes, the other fields have long come into thermal equilibrium and do not remember anything about their past.

As long as $\langle\varphi\rangle \ll M$ one can use the linear approximation to compute the expectation value of $\varphi[9]$ :

$$
\langle\varphi(x)\rangle \simeq \int d^{4} x \Delta_{\text {ret }}\left(x-x^{\prime}\right) j\left(x^{\prime}\right)
$$

with the retarded propagator

$$
\Delta_{\text {ret }}\left(x-x^{\prime}\right) \equiv\left\langle\varphi(x) \varphi\left(x^{\prime}\right)\right\rangle_{\text {ret }} \equiv i \Theta\left(t-t^{\prime}\right)\left\langle\left[\varphi(x), \varphi\left(x^{\prime}\right)\right]\right\rangle_{\mathrm{eq}} .
$$

Here the expectation value is taken in a thermal ensemble with the initial temperature ${ }^{3}$. The Fourier transform of the retarded propagator has an analytic continuation

$$
\Delta(p)=\frac{1}{-p^{2}+m^{2}+\Pi(p)}
$$

into the upper half of the complex frequency plane such that

$$
\Delta_{\text {ret }}(p)=\Delta\left(p^{0}+i \epsilon, \boldsymbol{p}\right) \text {. }
$$

We are interested in spatially homogeneous oscillations and can therefore consider an $\boldsymbol{x}$-independent $j$. Without any interaction $\varphi$ would simply oscillate with the frequency $m$. With interaction, $m^{2}$ receives finite temperature corrections given by the real part of $\Pi$. The imaginary part of $\Pi$ leads to exponential damping of the $\varphi$ oscillations. We assume that the damping rate is much smaller than the oscillation frequency, which will be verified a posteriori ${ }^{4}$. Then the damping rate is given by

$$
\gamma=-\frac{1}{2 m_{T}} \operatorname{Im} \Pi\left(m_{T}+i \epsilon, \mathbf{0}\right)
$$

\footnotetext{
${ }^{3}$ The system may get heated by applying the external field, but this effect does not contribute in the linear approximation.

${ }^{4}$ Yokoyama [7] finds a damping rate which is much larger than the frequency.
} 
where $m_{T}$ is the thermally corrected frequency. Since $m_{T}$ is much smaller than all other relevant energy scales in the problem, we can write

$$
\gamma \simeq-\lim _{\omega \rightarrow 0} \frac{1}{2 \omega} \operatorname{Im} \Pi(\omega+i \epsilon, \mathbf{0})
$$

This limit exists since $\Pi$ is real at zero frequency. Therefore, at leading order in $m / T$, the damping rate does not depend on $m^{5}$. For large $T$ one can neglect the masses of the matter particles so that, by dimensional analysis,

$$
\gamma=f \frac{T^{3}}{M^{2}}
$$

where $f$ is a function of the (dimensionless) gauge, Yukawa, and scalar couplings. For $T \lesssim T_{\text {osc }}$ we have $\gamma \lesssim m f \sqrt{m / M}$, which is indeed much smaller than $m$.

In general the calculation of $f$ is non-trivial. We consider a simple model [7] where $\varphi$ is coupled to a massless scalar field $\chi$, which mimics a Standard Model field. We will see that for this case the damping rate can be expressed through the bulk viscosity, which has been computed elsewhere. The Lagrangian is

$$
\mathcal{L}=\mathcal{L}_{\varphi}+\mathcal{L}_{\chi}+\mathcal{L}_{\varphi \chi}
$$

with

$$
\begin{gathered}
\mathcal{L}_{\varphi}=\frac{1}{2}(\partial \varphi)^{2}-\frac{m^{2}}{2} \varphi^{2} \\
\mathcal{L}_{\chi}=\frac{1}{2}(\partial \chi)^{2}-\frac{\lambda}{4 !} \chi^{4} \\
\mathcal{L}_{\varphi \chi}=\frac{g^{2}}{2 M} \varphi(\partial \chi)^{2}
\end{gathered}
$$

The coupling constant $\lambda$ is supposed to be reasonably large, so that the field $\chi$ is in thermal equilibrium, but small enough so that perturbation theory can be applied. At leading order in $M^{-1}$ the $\varphi$ self-energy can be written as

$$
\Pi\left(p^{0}+i \epsilon, \boldsymbol{p}\right)=-\frac{g^{4}}{4 M^{2}} \int d^{4} x e^{i p \cdot x}\left\langle(\partial \chi)^{2}(x)(\partial \chi)^{2}(0)\right\rangle_{\text {ret }},
$$

\footnotetext{
${ }^{5}$ In Ref. [7] an $m$-dependent damping rate was obtained. One cause for this discrepancy appears to be that in going from Eq. (21) to Eq. (22) of Ref. [7] the $\chi$-damping rate $\Gamma_{p}$ has been neglected in favour of $m$, even though $\Gamma_{p} \gg m$.
} 
where the 2-point function in Eq. (12) has to be evaluated for $M \rightarrow \infty$. In this limit the operator $(\partial \chi)^{2}$ is related to the trace of the energy momentum tensor of $\chi$

$$
T^{\mu \nu} \equiv \partial^{\mu} \chi \partial^{\nu} \chi-\eta^{\mu \nu} \mathcal{L}_{\chi}
$$

through the trace anomaly [1],

$$
T_{\mu}^{\mu}=-\frac{\beta(\lambda)}{4 \lambda}(\partial \chi)^{2}+\cdots
$$

Here $\beta(\lambda)=3 \lambda^{2} /\left(16 \pi^{2}\right)+O\left(\lambda^{3}\right)$ is the $\beta$ function of the scalar $\chi^{4}$ theory. The ellipsis in Eq. (14) denotes total derivatives and terms which vanish by the equations of motion, neither of which contribute to the imaginary part of $\Pi$ in the limit (6). Combining Eqs. (6), (12), and (14), we obtain at leading order in $\lambda$

$$
\gamma=\frac{g^{4}}{8 M^{2}} \frac{(8 \pi)^{4}}{9 \lambda^{2}} \lim _{\omega \rightarrow 0} \frac{1}{\omega} \operatorname{Im} \int d^{4} x e^{i \omega x^{0}}\left\langle T_{\mu}^{\mu}(x) T_{\nu}^{\nu}(0)\right\rangle_{\mathrm{ret}} .
$$

The right-hand side is related to the bulk viscosity $\zeta$ of a gas of $\chi$ particles through the Kubo relation [12]

$$
\zeta=\frac{1}{9} \lim _{\omega \rightarrow 0} \frac{1}{\omega} \operatorname{Im} \int d^{4} x e^{i \omega x^{0}}\left\langle T_{\mu}^{\mu}(x) T_{\nu}^{\nu}(0)\right\rangle_{\mathrm{ret}} .
$$

We thus find that the damping rate of the moduli field $\varphi$ in this model is directly proportional to the bulk viscosity of the plasma

$$
\gamma=\frac{g^{4}}{8 M^{2}} \frac{(8 \pi)^{4}}{\lambda^{2}} \zeta
$$

In order to compute $\zeta$ even at leading order in $\lambda$ one has to sum an infinite set of diagrams [12. This turns out to be equivalent to solving an appropriate Boltzmann equation, which accounts for particle-number-changing processes. The result is [13]

$$
\zeta=\frac{b}{6(32 \pi)^{4}} \lambda \ln ^{2}(\xi \lambda) T^{3}
$$

where $b=5.5 \times 10^{4}$ and $\xi=\exp \left(15 \zeta(3) / \pi^{2}\right) / 96=0.064736$. Then we finally obtain

$$
\gamma \simeq 4.5 g^{4} \frac{\ln ^{2}(\xi \lambda)}{\lambda} \frac{T^{3}}{M^{2}}
$$

for the $\varphi$ damping rate in the model (8) . 
We have thus seen that the damping rate of moduli fields in a thermal environment is parametrically larger than in the vacuum, when $T \gtrsim m$. However, it is always small compared to the Hubble rate. Therefore thermal effects alone cannot solve the cosmological moduli problem.

Acknowledgements I would like to thank M. Ratz for bringing Ref. [7] to my attention, W. Buchmüller, S. Huber, and M. Laine for useful comments, and the referee for pointing out a numerical error in an earlier version of this paper. This work was supported in part through the DFG funded Graduate School GRK 881.

\section{References}

[1] G. D. Coughlan, W. Fischler, E. W. Kolb, S. Raby and G. G. Ross, Cosmological problems for the Polonyi potential, Phys. Lett. B 131 (1983) 59.

[2] A. S. Goncharov, A. D. Linde and M. I. Vysotsky, Cosmological problems for spontaneously broken supergravity, Phys. Lett. B 147 (1984) 279.

[3] G. German and G. G. Ross, A cosmological problem for maximally symmetric supergravity, Phys. Lett. B 172 (1986) 305;

[4] J. R. Ellis, D. V. Nanopoulos and M. Quiros, On the axion, dilaton, Polonyi, gravitino and shadow matter problems in supergravity and superstring models, Phys. Lett. B 174 (1986) 176.

[5] T. Banks, D. B. Kaplan and A. E. Nelson, Cosmological implications of dynamical supersymmetry breaking, Phys. Rev. D 49 (1994) 779 arXiv:hep-ph/9308292.

[6] L. Randall and S. D. Thomas, Solving the cosmological moduli problem with weak scale inflation, Nucl. Phys. B 449 (1995) 229 arXiv:hep-ph/9407248.

[7] J. Yokoyama, Thermal background can solve the cosmological moduli problem, arXiv:hep-ph/0601067.

[8] A. D. Linde, Relaxing the cosmological moduli problem, Phys. Rev. D 53 (1996) 4129 arXiv:hep-th/9601083.

[9] L.D. Landau, E.M. Lifshitz, Statistical Mechanics (Pergamon Press, Oxford, 1980). 
[10] See, for instance, [12].

[11] J. C. Collins, The energy - momentum tensor revisited, Phys. Rev. D 14 (1976) 1965.

[12] S. Jeon, Hydrodynamic transport coefficients in relativistic scalar field theory, Phys. Rev. D 52 (1995) 3591 arXiv:hep-ph/9409250.

[13] S. Jeon and L. G. Yaffe, From quantum field theory to hydrodynamics: transport coefficients and effective kinetic theory, Phys. Rev. D 53 (1996) 5799 arXiv:hep-ph/9512263. 